\title{
Semantic Encoding and Retrieval in the Left Inferior Prefrontal Cortex: A Functional MRI Study of Task Difficulty and Process Specificity
}

\author{
Jonathan B. Demb, ${ }^{1}$ John E. Desmond, ${ }^{2}$ Anthony D. Wagner, ${ }^{1}$ Chandan J. Vaidya, ${ }^{1}$ Gary H. Glover, ${ }^{2}$ and \\ John D. E. Gabrieli ${ }^{1}$
}

Departments of ${ }^{1}$ Psychology and ${ }^{2}$ Radiology, Stanford University, Stanford, California 94305-2130

Prefrontal cortical function was examined during semantic encoding and repetition priming using functional magnetic resonance imaging (fMRI), a noninvasive technique for localizing regional changes in blood oxygenation, a correlate of neural activity. Words studied in a semantic (deep) encoding condition were better remembered than words studied in both easier and more difficult nonsemantic (shallow) encoding conditions, with difficulty indexed by response time. The left inferior prefrontal cortex (LIPC) (Brodmann's areas $45,46,47)$ showed increased activation during semantic encoding relative to nonsemantic encoding regardless of the relative difficulty of the nonsemantic encoding task. Therefore, LIPC activation appears to be related to semantic encoding and not task difficulty. Semantic encoding decisions are performed faster the second time words are presented. This represents semantic repetition priming, a facilitation in semantic processing for previously encoded words that is not dependent on intentional recollection. The same LIPC area activated during semantic encoding showed decreased activation during repeated semantic encoding relative to initial semantic encoding of the same words. This decrease in activation during repeated encoding was process specific; it occurred when words were semantically reprocessed but not when words were nonsemantically reprocessed. The results were apparent in both individual and averaged functional maps. These findings suggest that the LIPC is part of a semantic executive system that contributes to the on-line retrieval of semantic information.

[Key words: functional magnetic resonance imaging (fMRI), prefrontal cortex, semantic memory, implicit memory, area 8 , executive functioning]

Encoding and retrieval constitute two discrete stages of memory. Encoding refers to the processes operating at the time of learning that determine what information is stored in long-term memory. Different kinds of encoding lead to different kinds of storage. For example, words are typically better remembered when encoded for meaning (semantic or "deep" encoding) rather than for appearance (nonsemantic or "shallow" encoding) (Craik and

Received Jan. 17, 1995; revised May 5, 1995; accepted May 10, 1995.

This work was supported by research grants from the Stanford University Office of Technology and Licensing, the Alzheimer's Association (Blum-Kovler Pilot Research Grant), and the NIH (NINDS 1F32NSO9628).

Correspondence should be addressed to Jonathan Demb at the above address. Copyright (c) 1995 Society for Neuroscience $\quad 0270-6474 / 95 / 155870-09 \$ 05.00 / 0$
Lockhart, 1972). Retrieval refers to the recovery of previously encoded information from long-term memory and can be measured explicitly, by measures of intentional recollection, or implicitly, by measures of priming, conditioning, or skill learning (Graf and Schacter, 1985). Priming reflects experience-based facilitation in performance with a stimulus that does not depend on intentional recollection. For example, subjects make a semantic encoding decision (e.g., is this word abstract?) more quickly for a repeated than an initial presentation of a word (Gabrieli, Desmond, Demb, Wagner, Stone, Vaidya, and Glover, unpublished observations).

Functional magnetic resonance imaging (fMRI) and positron emission tomography (PET) studies have indicated left inferior prefrontal cortex (LIPC) involvement in semantic encoding. The LIPC area (Brodmann's areas 45, 46, 47), anterior to Broca's area, shows greater activation during semantic than nonsemantic encoding (Gabrieli et al., unpublished observations; see Tulving et al., 1994). In these studies, the semantic encoding tasks have been more difficult than nonsemantic encoding tasks, with difficulty operationalized as the time required to perform an encoding task. This difference in difficulty has been shown directly in two studies (Gabrieli et al., unpublished observations; Kapur et al., 1994) and is likely to be the case in others. One goal of the current study was to compare a semantic encoding task to both less and more difficult nonsemantic encoding tasks, to investigate whether LIPC activation reflects semantic encoding or encoding difficulty. If LIPC activation reflects semantic encoding, it should be present during the semantic encoding task relative to both less and more difficult nonsemantic encoding tasks.

The same LIPC area that activates during semantic encoding has also been implicated in scmantic repctition priming. When words were semantically encoded more than once, subjects encoded more quickly the second time (priming). There was a corresponding decrease in LIPC activation for repeated relative to initial semantic encoding (Gabrieli et al., unpublished observations; Raichle et al., 1994). The decreases in response time and brain activation represent a word-specific effect; when novel words were semantically encoded, behavioral responses and LIPC activation returned to initial levels (Gabrieli et al, unpublished observations; Raichle et al., 1994). However, it is unknown whether decreased LIPC activation reflects a processspecific change based on semantic reencoding, or rather a process nonspecific change based on reexposure to words regardless of the encoding process engaged. Therefore, a second goal was to clarify the nature of the decreased LIPC activation during repeated semantic encoding. If decreased LIPC activation is spe- 


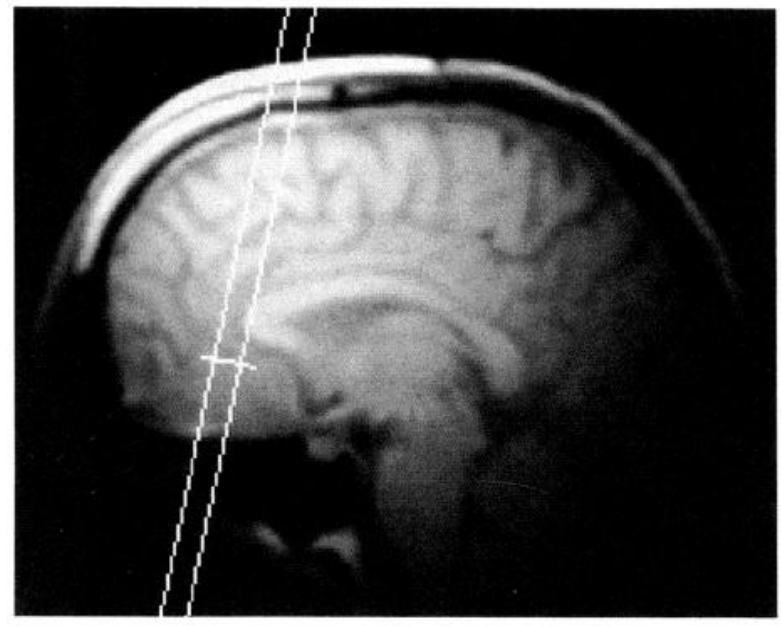

Figure 1. The location of the coronal slices analyzed in this study are depicted as white lines on a sagittal localizer. The slices shown are 32 and $39 \mathrm{~mm}$ rostral to the anterior commissure $(A C)$. An additional third slice, $46 \mathrm{~mm}$ rostral to the $\mathrm{AC}$ was acquired in three subjects in the Process Specificity study (see Table 1).

cific to semantic processes, it should occur during repeated semantic encoding but not during repeated nonsemantic encoding.

\section{Materials and Methods}

\section{Behavioral study}

The purpose of the behavioral study was to validate a difficult nonsemantic encoding condition to be used in the Task Difficulty fMRI experiment. We predicted that this task would be more difficult than semantic encoding (longer response time; RT), but would still lead to lower subsequent memory performance than semantic encoding.

Subjects. The subjects were 16 volunteers from the Stanford community (ages 18-24) who participated for a $\$ 10$ payment. All subjects gave informed consent.

Materials. The stimuli were taken from a set of 240 abstract and 240 concrete 3-8-letter-long words. Four word lists, each containing four blocks of 20 words each, were constructed with the constraint that in each block 10 words were abstract (e.g., "LOVE"), 10 words were concrete (e.g., "book"), 10 words were printed in uppercase letters, and 10 words were printed in lowercase letters. In addition, 10 words in each list had an ascending alphabetic relation between the first and last letter (e.g., "CAR", because "C" comes before "R" alphabetically), and 10 words had a descending relation (e.g., "hope," because "h" comes after " $\mathrm{e}$ " alphabetically). Words in each set appeared in a pseudorandom order with the constraints that no more than four abstract or concrete words, no more than four uppercase or lowercase words, and no more than four ascending or descending words appeared consecutively.

Procedure. Each subject performed two encoding experiments. Both experiments compared a semantic encoding task, in which subjects had to decide whether words were abstract or concrete (Abstract/Concrete Task), with a nonsemantic encoding task. In the first experiment, subjects alternately performed the semantic encoding task and a nonsemantic encoding task, in which subjects had to decide if words were printed in uppercase or lowercase letters (Uppercase/Lowercase Task). In the second experiment, subjects alternately performed the semantic encoding task and a nonsemantic encoding task, in which subjects had to decide if the first and last letter of each word were ascending or descending alphabetically (Ascending/Descending Task). The semantic encoding task required that subjects attend to word meaning, whereas both nonsemantic encoding tasks required that subjects attend to word features (i.e., letters).

Subjects saw four sets of 20 words presented consecutively. One encoding task was performed on the first and third set and another task was performed on the second and fourth set. Each pair of tasks constituted a cycle, so that two cycles were performed by each subject in each experiment. An instruction card at the beginning of each set of 20 words (e.g., Task-Abstract) indicated the target for that set (e.g., abstract words). Subjects were instructed to respond as quickly and as accurately as possible to the indicated target by pressing a mouse button; they were instructed to not respond to nontarget words. Words appeared centrally on a computer monitor for a duration of $1 \mathrm{sec}$, followed by a 1 sec interval before the next word appeared. For each task, half of the subjects responded to one kind of target (e.g., abstract or uppercase or ascending) and half the subjects responded to the other kind of target (e.g., concrete or lowercase or descending). Word orders were kept constant across subjects, but the order of alternating encoding tasks was counterbalanced across subjects. Thus, encoding tasks and responses were counterbalanced across words. After each experiment, subjects were given a recognition test, composed of 20 studied words, half of which were presented in the Abstract/Concrete Task and half of which were presented in either the Uppercase/Lowercase Task or the Ascending/Descending Task, and 20 new (foil) words in a random order. Words appeared one at a time on a computer monitor and subjects were instructed to respond by pressing a mouse button if they thought the words had been in the study list. Different foil words were used after each encoding experiment, and all words appeared as studied words and foil words equally often.

\section{fMRI study}

Subjects. The subjects were 10 right-handed volunteers (9 males) from the Stanford community (ages 23-31) who participated for a $\$ 20-30$ payment. Four subjects were scanned in the Task Difficulty Experiment, and six subjects were scanned in the Process Specificity Experiment. All subjects gave informed consent.

Materials. The tasks and materials in the fMRI study were used from the encoding tasks described above with two changes. First, subjects performed each task with eight alternating sets of words (four cycles) instead of four alternating sets of words (two cycles). Second, subjects

Table 1. Experimental design

\begin{tabular}{llll} 
Experiment & Task comparisons & Cognitive comparisons & Data acquisition \\
\hline Task difficulty & Abstract/Concrete vs & Semantic Encoding vs & Two single slices (four subjects) \\
& Uppercase/Lowercase & Easy Nonsemantic Encoding & \\
& Abstract/Concrete vs & Semantic Encoding vs & \\
Ascending/Descending & Difficult Nonsemantic Encoding & Two single slices (two subjects) \\
Process specificity & Abstract/Concrete (i) vs & Initial Semantic Encoding & Multi three-slice (three subjects) \\
& Abstract/Concrete (r) & vs Repeated Semantic Encoding & Multi two-slice (one subject) \\
& Uppercase/Lowercase (i) vs & (Semantic Repetition Priming) & Initial Nonsemantic Encoding \\
Uppercase/Lowercase (r) & vs Repeated Nonsemantic Encoding & (Nonsemantic Repetition Priming)
\end{tabular}

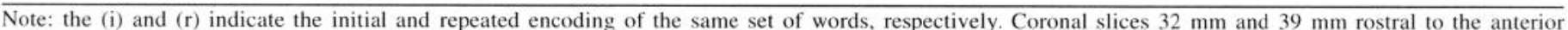

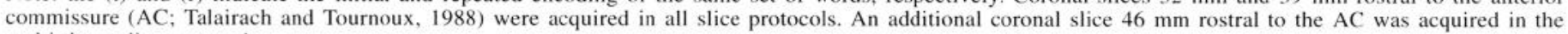
multi three-slice protocol. 
a

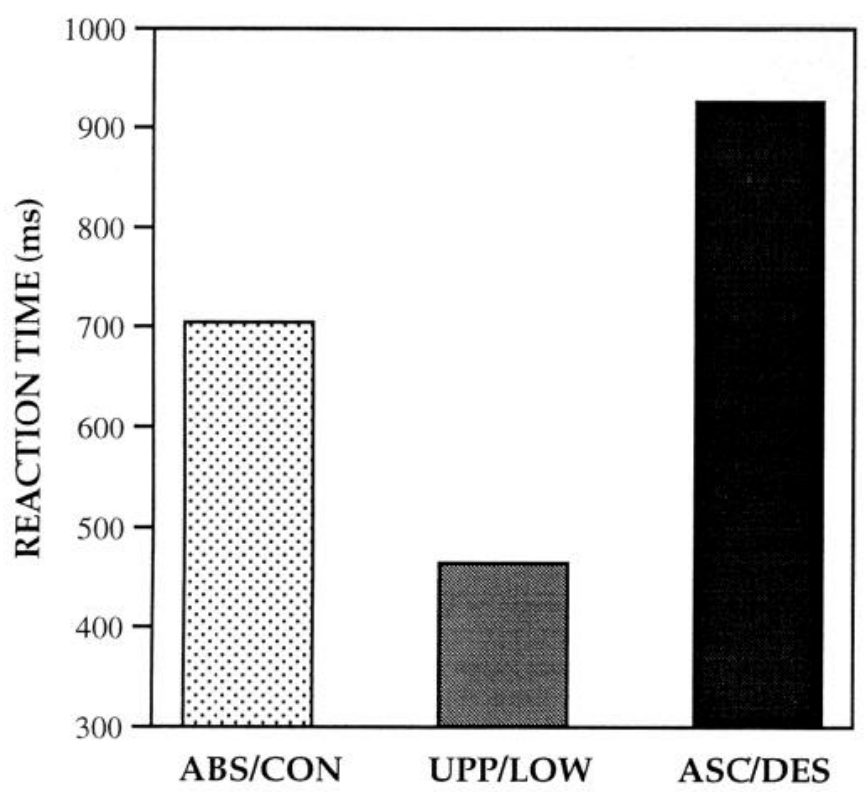

ENCODING TASK b

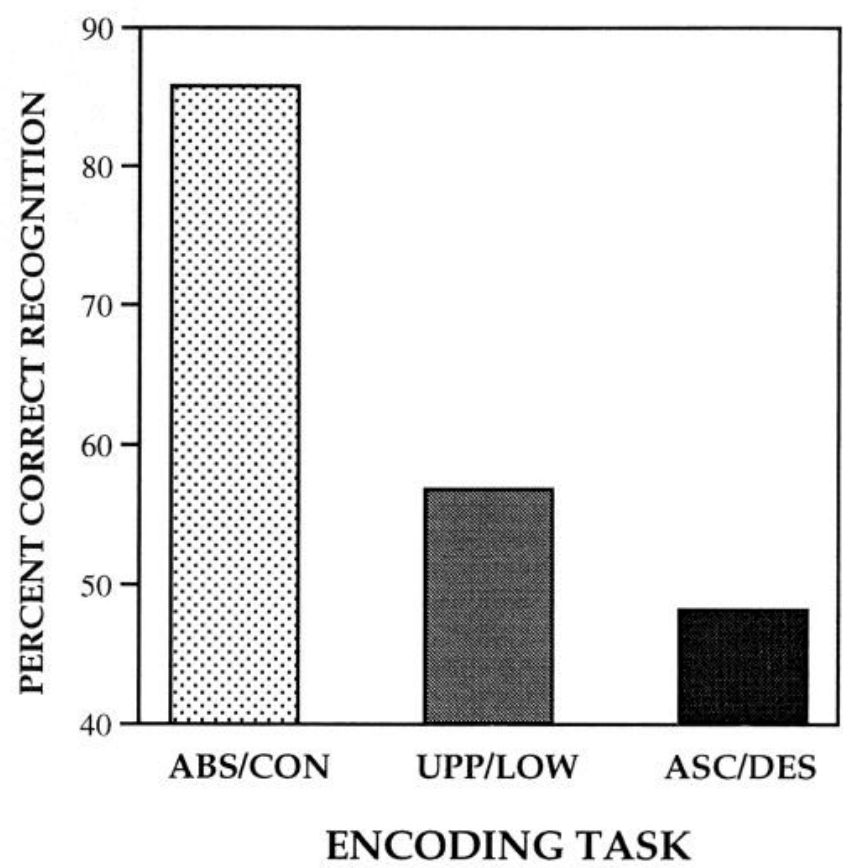

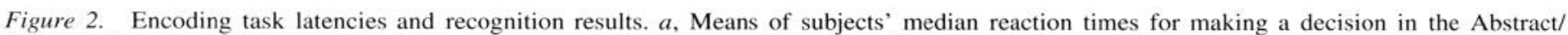

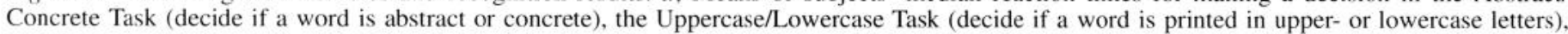

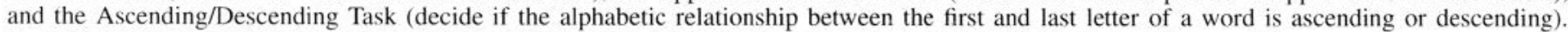
$b$, Mean percentages of corrected recognition (hits + correct rejections) for words studied in the three encoding conditions.

responded by pressing a pneumatic bulb rather than by pressing a mouse button. Across subjects, encoding tasks and responses (e.g., abstract or concrete) were counterbalanced across words as described above. In the Task Difficulty Experiment, different words appeared in each of the eight word sets. In the Process Specificity Experiment, four unique word sets were presented a first time (sets 1,3,5, and 7) and then a second time (sets $2,4,6$, and 8 ) in different orders (e.g., set 2 was a reordering of set 1 ).

Procedure. Imaging was performed with a $1.5 \mathrm{~T}$ whole-body MRI scanner (General Electric Medical Systems Signa, Rev. 5.3). For functional imaging, two 5 inch diameter local receive coils were used for signal amplification. Head movement was minimized by using a "bitebar" formed with each subject's dental impression. A T2* sensitive gradient echo spiral sequence (Meyer et al., 1992), which is relatively insensitive to cardiac pulsatility motion artifacts (Noll et al., in press), was used for functional imaging with parameters of TR $=75 \mathrm{msec}$, TE $=40 \mathrm{msec}$, and flip angle $=23^{\circ}$. Twenty interleaves were obtained for each image, with a total acquisition time of $1.5 \mathrm{sec}$ per image. In the Task Difficulty Experiment, two $7 \mathrm{~mm}$ thick slices (in-plane resolution of $2.4 \times 2.4 \mathrm{~mm}$ ) were acquired separately in the coronal plane of Talairach and Tourneaux (1988) at $32 \mathrm{~mm}$ and $39 \mathrm{~mm}$ rostral to the anterior commissure (AC) (see Fig. 1). In the Process Specificity Experiment, the same two slices were acquired in all six subjects; an additional third slice, $46 \mathrm{~mm}$ rostral to the $\mathrm{AC}$, was also acquired in three subjects.

In the Task Difficulty Experiment, fMRI measurements were acquired from one slice at a time, and this procedure was also used for two of the subjects in the Process Specificity Experiment. For each of the recordings, 224 images were acquired continuously every $1.5 \mathrm{sec}$ over a $336 \mathrm{sec}$ session. For the other four subjects in the Process Specificity Experiment, a multislice sequence was used. For this sequence, a slice at each prescribed location was obtained in a repeating order. So, for example, if three slices were prescribed, slice acquisition would precede as $1,2,3,1,2,3$, etc. With a constant slice acquisition rate of one every $1.5 \mathrm{sec}$, the temporal spacing of a given slice in an $n$ slice acquisition was $n \times 1.5 \mathrm{sec}$. For three subjects in the Process Specificity Experiment, a multi 3 -slice acquisition was obtained so that each slice contained 75 data points at a $4.5 \mathrm{sec}$ temporal spacing. For one subject in the Process Specificity Experiment, a multi 2-slice acquisition was obtained so that each slice contained 112 data points at a $3 \mathrm{sec}$ temporal spacing (see Table 1). T1-weighted, flow compensated spin-warp anatomy images $(\mathrm{TR}=500 \mathrm{msec}$, minimum TE) were acquired for all sections that received functional scans, and pixels found to be significantly activated during the functional scan were overlaid on these structural images.

For subjects in the Task Difficulty Experiment, scanning was performed separately during two conditions. In the first condition, they performed the Abstract/Concrete Task or the Uppercase/Lowercase Task on alternating sets of words (four cycles), and in the second condition they performed the Abstract/Concrete Task or the Ascending/Descending Task on alternating sets of words (four cycles) (Table 1). The two conditions were performed in that order, and recordings were made first in the posterior slice and then in the anterior slice.

Subjects in the Process Specificity Experiment were also scanned

Figure 3. Individual fMRI activations for one subject in the Task Difficulty Experiment and one subject in the Process Specificity Experiment. Greater fMRI activation during semantic encoding (Abstract/Concrete Task) relative to easy nonsemantic encoding (Uppercase/Lowercase Task) (upper row, left side) and during semantic encoding relative to difficult nonsemantic encoding (Ascending/Descending Task) (upper row, right side). Decreased fMRI activation during repeated relative to initial semantic encoding (semantic repetition priming) (lower row, left side) and during repeated relative to initial nonsemantic encoding (nonsemantic repetition priming) (lower row, right side). Left side of image corresponds to left side of brain. Slices from both subjects are coronal sections. $39 \mathrm{~mm}$ rostral to the AC (Talairach and Tournoux, 1988). The color scale indicates all $r$ values between the minimum $r$ threshold needed to reach statistical significance ( $p<0.01$, one-tailed) (min) and the maximum $r$ value in that slice $(\max )$. 


\section{Individual Functional Activations}

\section{Task Difficulty}

Semantic Encoding

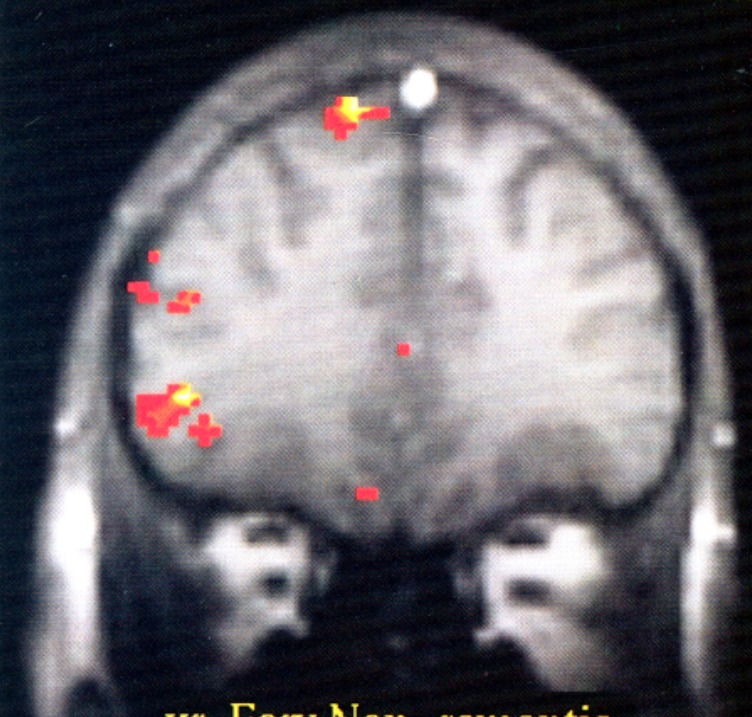

vs. Easy Non-semantic

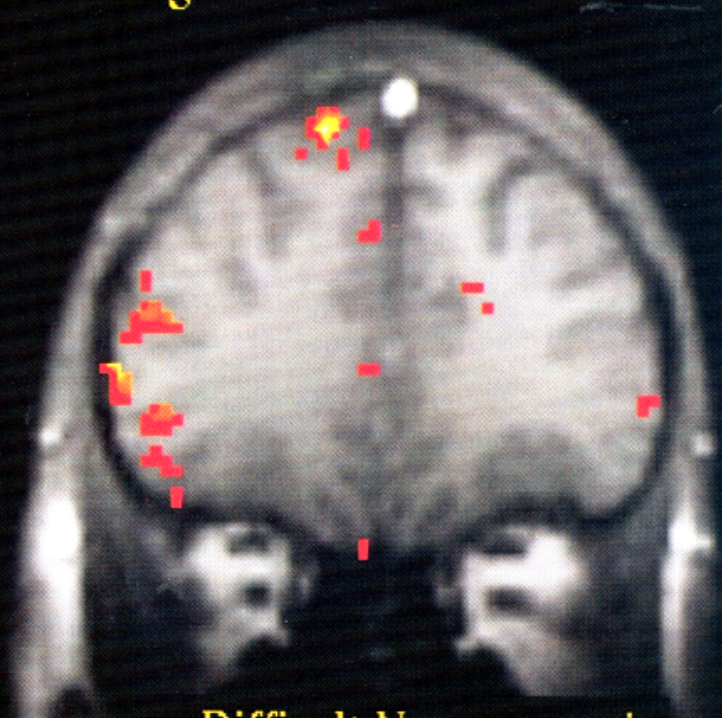

vs. Difficult Non-semantic

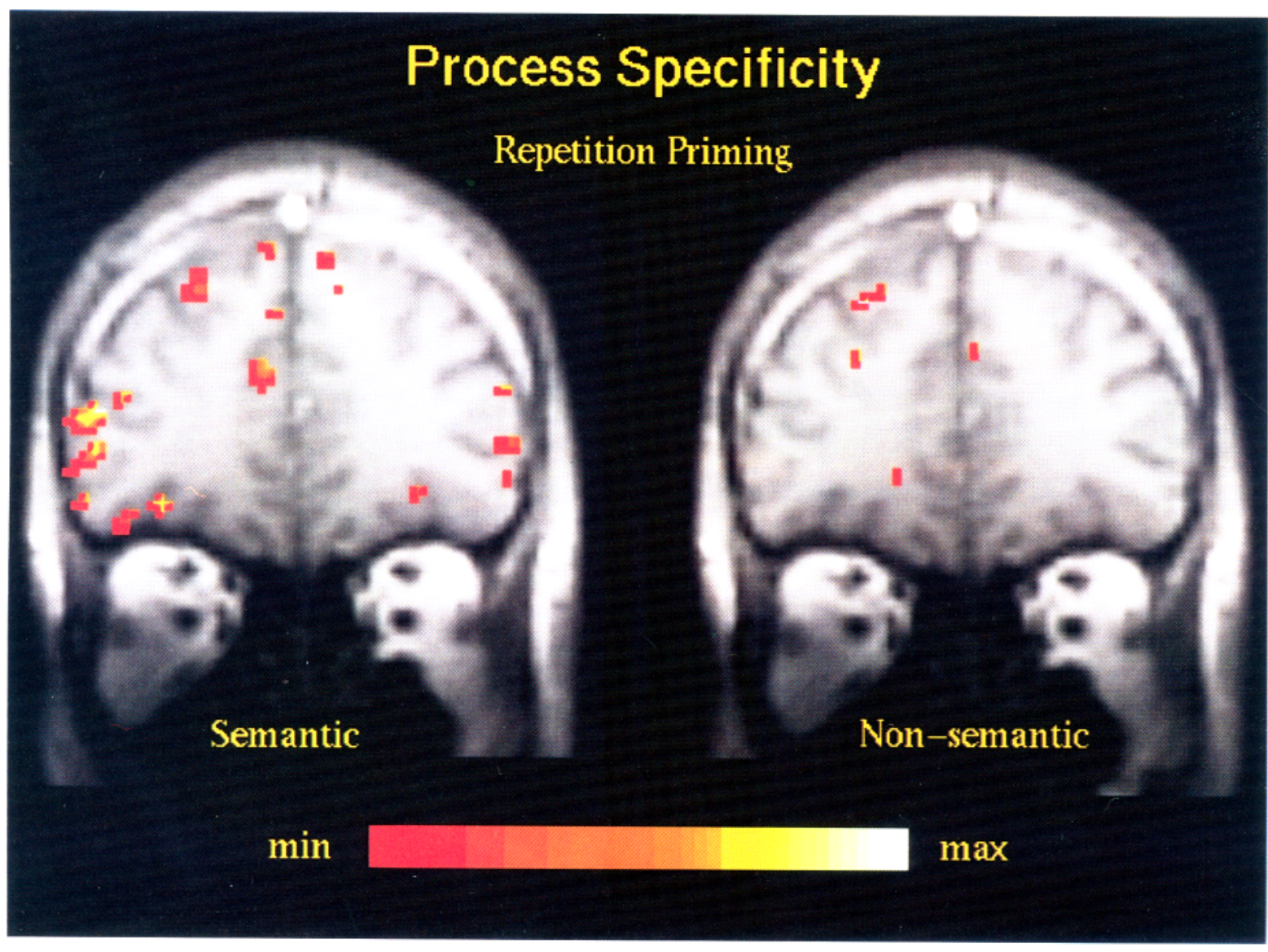


during two conditions. In the first condition, they performed the $\mathrm{Ab}$ stract/Concrete Task on initially presented and repeated words (four cycles), and then they performed the Uppercase/Lowercase Task on initially presented and repeated words (Four cycles) (Table 1). Subjects scanned in the single slice protocol performed the experiments separately in both slices as described above. Subjects scanned in the multislice protocols performed each experiment only once.

Stimuli were generated from a computer and back-projected onto a screen located above the subject's neck via a magnet-compatible projector; visual images were viewed from a mirror mounted above the subject's head. Subjects were asked to respond by pressing a pneumatic bulb in response, to either concrete or abstract words during the Abstract/Concrete Task, to either upper or lower case words during the Uppercase/Lowercase Task, and to either ascending or descending words during the $\Lambda$ scending/Descending Task.

Data analysis. Image reconstruction was performed off-line by transferring the raw data to a Sun SparcStation. A gridding algorithm was employed to resample the raw data into a Carlesian matrix prior to processing with $2 \mathrm{~d}$ FFT. Once individual images were reconstructed, time-series of each pixel were obtained and cross-correlation methods that take advantage of periodically oscillating paradigms were used to analyze functional activation. Because a considerable amount of artifactual signal that occurs over time is due to events that are random with respect to the timing of the activation paradigm (e.g., pulsatile effects from blood, CSF, or brain movement), cross-correlations of the pixel responses over time with a reference function that represents the timing of the expected activation (based upon the timing of stimulus presentation) can be used to remove artifacts (Bandettini et al., 1993; Lee et al., in press). Analyses were performed by first removing linear slopes that occurred over the entire scan. The Pearson correlation of the time-series for each pixel was then computed with respect to a sinusoidal reference waveform. The same reference waveform was used for each pixel. The frequency of the reference function was computed from the number of task cycles divided by the total time of the experiment. For the present study, one task cycle consisted of either a block of semantic and a block of nonsemantic encoding (Task Difficulty Experiment) or a block of initial and a block of repeated encoding (Process Specificity Experiment). Because four of these cycles were presented over a $336 \mathrm{sec}$ scan, the target frequency was $\sim 0.012 \mathrm{~Hz}$. A correlation with a sine $\left(r_{s}\right)$ and with a cosine $\left(r_{c}\right)$ function was computed, which allows the temporal phase of the correlated time-series to be computed by the expression

$$
\phi_{r}=\tan ^{-1}\left(\frac{r_{s}}{r_{c}}\right)
$$

and the correlation magnitude by the expression

$$
r_{m}=\sqrt{r_{s}^{2}+r_{c}^{2}}
$$

To construct functional activation maps, pixels overlaying the brain that satisfied the criteria of: (1) $r_{m}>T$, where $T$ is the threshold for statistical significance at $p<0.01$ (one-tailed) (for single slice protocol with $N$ $=224, T=0.155$, for the multi 2 -slice protocol with $N=112, T=$ 0.220 , and for the multi 3 -slice protocol with $N=75, T=0.268$ ). In one slice of one subject, there was a source of artifact (e.g., head motion) in the first task cycle, so the correlations were recomputed with the last thee cycles $(N-168)$ using an adjusted $r$ threshold $(T=$ 0.171 ); and (2) phase lag in, or within 2 standard errors of, an estimated 3-9 sec hemodynamic delay window (with a cycle duration of $84 \mathrm{sec}$, $3-9 \mathrm{sec}$ corresponds to $12.9-38.6^{\circ}$ ). 'I he standard deviation of the phase in radians can be calculated as follows (Lee et al., in press):

$$
\sigma_{\phi} \approx \frac{\left(1-r_{m}^{2}\right)}{r_{m}^{2}} \frac{1}{\sqrt{N-3}}
$$

From this expression, the standard error of the phase was estimated to be approximately $10.4^{\circ}$ in all experiments. Thus, a phase range of $-8^{\circ}$ to $+59^{\circ}$ (with respect to a sine wave) was used in conjunction with criterion (1) to select functionally relevant pixels. This phase range was used to image correlated pixels in the parenchyma that responded a few seconds after task onset. Correlated pixels with longer phase lags tended to appear in larger vessels consistent with the notion that a lag in temporal response is characteristic of larger, draining veins (Lee et al., in press). This map was processed with a median filter with spatial width $=2$ to emphasize spatially coherent patterns of activation. The filter was used based on the assumption that spuriously correlated pixels (i.e., false positives due to type I errors) are less likely to occur in clusters than genuinely correlated pixels, and thus clusters of correlated pixels are more likely to reflect an active region. The resulting map was overlaid on a T1-weighted structural image. For the Task Difficulty Experiment, the phase lag window chosen reflected pixel values that increased during the Abstract/Concrete Task and decreased during either the Uppercase/Lowercase Task or the Ascending/Descending Task. [This phase window identifies pixels that increase during the Abstract/Concrete Task and decrease during the control task only when the Abstract/Concrete Task occurs first. Because order of task presentation was counterbalanced, a phase window that was shifted by $180^{\circ}$ (i.e., $172^{\circ}$ to $239^{\circ}$ ) was used when the Abstract/Concrete Task occurred second.] For the Process Specificity Experiment, the phase lag window reflected pixels values that increased during initial and decreased during repeated encoding in the Abstract/Concrete Task or the Uppercase/Lowercase Task.

To obtain composite maps of activation over all subjects, averaged functional activation maps for both experiments were created by transforming the coronal sections 32 and $39 \mathrm{~mm}$ rostral to the $\mathrm{AC}$ from every subject to a common standardized coronal section (Talairach and Tourneaux, 1988), $35 \mathrm{~mm}$ rostral to the AC (see Desmond et al., 1995). Significant correlation values from each slice were transformed into a proportion of the maximum correlation value in that slice. This normalization allows for comparisons across slices and across subjects. Also, this normalization was necessary because the $r$ threshold varied depending on the functional protocol in the Process Specificity Experiment (see above). The averaged functional activation maps represent normalized correlation-weighted sums across subjects. The same color scale applies to all averaged images to allow for comparisons of correlation magnitude across experiments.

\section{Results}

\section{Behavioral study}

The mean of subjects' median reaction times were calculated for the three encoding tasks (Fig. 2). Initial analyses showed that response times in the Abstract/Concrete Task did not differ in the two encoding comparisons $[t(15)-0.26, p-0.80]$, so the data from this task were combined for subsequent analyses. A repeated measures analysis of variance (ANOVA) showed that response latencies differed among the three encoding conditions $[F(1,15)=115.52, p<0.0001]$. Subjects responded more quickly in the Uppercase/Lowercase Task $(M=465 \mathrm{msec}, \mathrm{SD}$ $=60)$ than in the Abstract/Concrete Task $(M=704 \mathrm{msec}, \mathrm{SD}$ $=56),[t(15)=12.79, p<0.0001]$ or the Ascending/Descending Task $(M=925 \mathrm{msec}, \mathrm{SD}=133),[t(15)=13.16, p<$ $0.0001]$. In addition, subjects responded more quickly in the Abstract/Concrete Task than in the Ascending/Descending Task $[t(15)=6.43, p<0.0001]$.

Percent correct on the recognition test (hits + correct rejections) was calculated for words studied in the three encoding tasks (Fig. 2). Initial analyses showed that percent correct for words studied in the Abstract/Concrete Task did not differ in the two encoding comparisons [ $t(15)-0.09, p-0.93]$, so data were combined in subsequent analyses. A repeated measures ANOVA showed that recognition performance differed for words studied in the three encoding conditions $[F(1,15)=$ $28.89, p<0.0001]$. Accuracy was higher for words encoded in the Abstract/Concrete Task $(M=85.8 \%, \mathrm{SD}=9.3 \%)$ than in the Uppercase/Lowercase Task $(M=56.9 \%, \mathrm{SD}=19 \%),[t(15)$ $=5.57, p<0.0001]$ or the Ascending/Descending Task $(M=$ $48.2 \%, S D=22.2 \%),[t(15)=7.91, p<0.0001]$. Recognition accuracy did not differ between the Uppercase/Lowercase and the Ascending/Descending Tasks $[t(15)=1.56, p>0.10]$.

\section{Imaging study}

In the Task Difficulty Experiment, all four subjects showed significant areas of increased LIPC activation during the Abstract/ Concrete Task relative to the Uppercase/Lowercase Task (Figs. 
3, 4; Semantic Encoding vs Easy Nonsemantic), consistent with our previous findings (Gabrieli et al., unpublished observations). Peak areas of activation in individual slices were most often in the left inferior frontal sulcus (LIFS) and in the left inferior frontal gyrus (LIFG), with correlation values as high as $r=$ 0.648 in the LIFS. In some slices, activation was observed also in area 8 of the left superior frontal gyrus (LSFG) and in area 10. Bilateral LIPC activation was present in some subjects, although in these cases the left-sided activation was usually stronger (i.e., higher correlations). The averaged functional map showed that the areas of highest activation occurred in regions of the LIFG and LIFS, corresponding to Brodmann's areas 45 , 46 , and 47 , as well as area 8 of the LSFG.

In the second comparison of the Task Difficulty Experiment, the same four subjects showed similar areas of increased LIPC activation during the Abstract/Concrete Task relative to the Ascending/Descending Task (Figs. 3, 4; Semantic Fncoding vs Difficult Nonsemantic). Individual slice activation was predominately found in the LIFS and LIFG, with peak activations as high as $r=0.722$ in the LIFS. Again, there was evidence of areas of activation in areas 8 and 10 . The overall pattern of activation appeared slightly more lateralized than it was in the previous comparison. The averaged functional map showed maximum overlap in the same LIPC regions described above (Brodmann's areas 45, 46, and 47), as well as area 8 of the LSFG.

In the Process Specificity Experiment, five of six subjects showed decreased activation in the LIPC during repeated rela tive to initial processing of words in the Abstract/Concrete Task (Figs. 3, 4; Semantic Repetition Priming), consistent with our previous findings (Gabrieli et al., unpublished observations). One subject (multi 3-slice protocol) did not show areas of activation in any comparison, and was dropped from further analyses. The other five subjects showed decreased areas of activation during repeated semantic encoding in the LIFG and LIFS (Brodmann's areas 45, 46, and 47), with magnitudes as high as $r=0.501$ in the LIFS. This pattern of activation was strongly left-lateralized with only a few cases of bilateral activation, and here the activation on the right side was always weaker than it was on the left. There were also areas of the LSFG (area 8) in four subjects that showed decreased activation during repeated encoding. The third slice ( $46 \mathrm{~mm}$ rostral to $\mathrm{AC}$ ) acquired in two of the five subjects showed a rostral extension of this dorsal activation. The averaged functional map showed the greatest overlaps in decreased activation during repeated semantic encoding in areas of the LIFG and LIFS, corresponding to Brodmann's arcas 45, 46, and 47. Also, therc was a considcrablc overlap in area 8 of the LSFG.

There were no consistent areas of decreased activation during repeated relative to initial nonsemantic encoding of words in the Uppercase/Lowercase Task (Figs. 3, 4; Nonsemantic Repetition Priming). The individual slices and the averaged functional map showed low magnitude correlations with no consistency across subjects.

\section{Discussion}

Task difficulty

The goal of the Task Difficulty study was to examine whether LIPC activation during semantic encoding observed in PET and fMRI studies reflects semantic processing or task difficulty. The critical comparison was a semantic encoding condition versus a more difficult nonsemantic encoding condition. If similar areas of LIPC show activation during semantic encoding relative to difficult nonsemantic encoding as well as easy nonsemantic encoding, then these areas probably correspond to the processes involved in semantic encoding rather than task difficulty.

The behavioral study confirmed that the Ascending/Descending Task was more difficult (i.e., took longer to perform) than the Abstract/Concrete Task, and that both of these tasks were more difficult than the Uppercase/Lowercase Task. Therefore, the Ascending/Descending Task represents difficult nonsemantic encoding and the Uppercase/Lowercase Task represents easy nonsemantic encoding. Recognition was better for words studied in the Abstract/Concrete Task than for words studied in either the Ascending/Descending Task or the Uppercase/Lowercase Task; words studied in the latter two conditions were remembered similarly. This confirms the finding that semantic encoding yields better recognition performance than nonsemantic encoding (Craik and I ockhart, 1972) even when nonsemantic encoding is more difficult than semantic encoding (Craik and Tulving, 1975).

The fMRI study demonstrated that the same LIPC area showed increased activation during semantic encoding relative to both an easier and a more difficult nonsemantic encoding task. This was evident in each individual subject and in the averaged functional activation map (Figs. 3, 4). The direct functional overlap between these two areas of activation strongly suggests that LIPC activation is related to the processes engaged in semantic encoding and not task difficulty. (A task difficulty explanation predicts greater LIPC activation for a more difficult nonsemantic task than a semantic task, the opposite of what was found.) Also, both comparisons showed semantic encoding activation in area 8 of the LSFG. This area may be part of a larger network that interacts with the LIPC during semantic processing (Demonet et al., 1992; Mazoyer et al., 1993).

The LIPC result has implications for other findings of activation during semantic processing. Several PET and fMRI studies have compared verb generation, in which subjects generate an appropriate verb to a given noun (e.g., cake-eat), with noun repetition. There was greater LIPC activation during verb generation than noun repetition (Petersen et al., 1988, 1989; McCarthy et al., 1993; Raichle et al., 1994). This comparison may be interpreted as a semantic/nonsemantic encoding comparison because words in the verb generation condition are subsequently remembered better than words in a noun repetition condition (Tulving et al., 1994), presumably because of the semantic elaboration required to produce a related word. However, generating verbs is also more difficult than simply reading nouns. The results of the present study suggest that activation during verb generation is related to semantic processes rather than task difficulty.

\section{Process specificity}

The goal of the Process Specificity study was to determine whether decreased LIPC activation during repeated semantic encoding of words (Gabrieli et al., unpublished observations) is process specific, that is, whether it reflect the repetition of semantic encoding processes or the mere repetition of words regardless of how the words are processed. The fMRI study showed decreased LIPC activation during repeated relative to initial semantic encoding of words, but there was no decreased LIPC activation during repeated relative to initial nonsemantic encoding of words. This was evident in five of six subjects and in the averaged functional activation map (Figs. 3, 4), suggesting 


\section{Averaged Functional Activations}

\section{Task Difficulty}

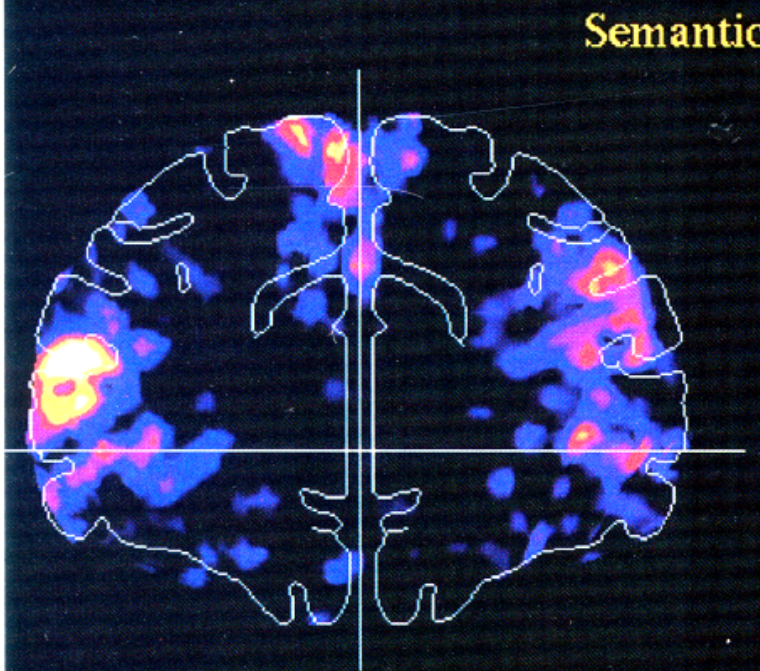

vs. Easy Non-semantic

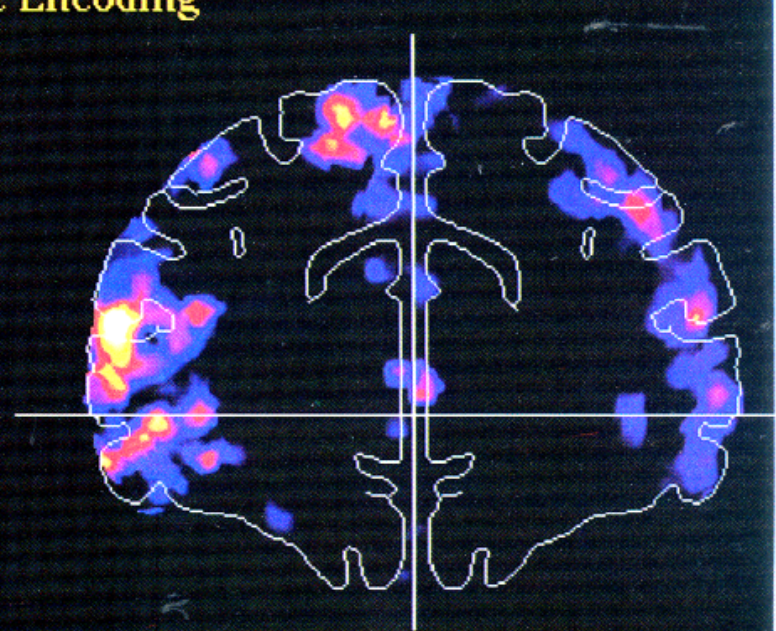

vs. Difficult Non-semantic

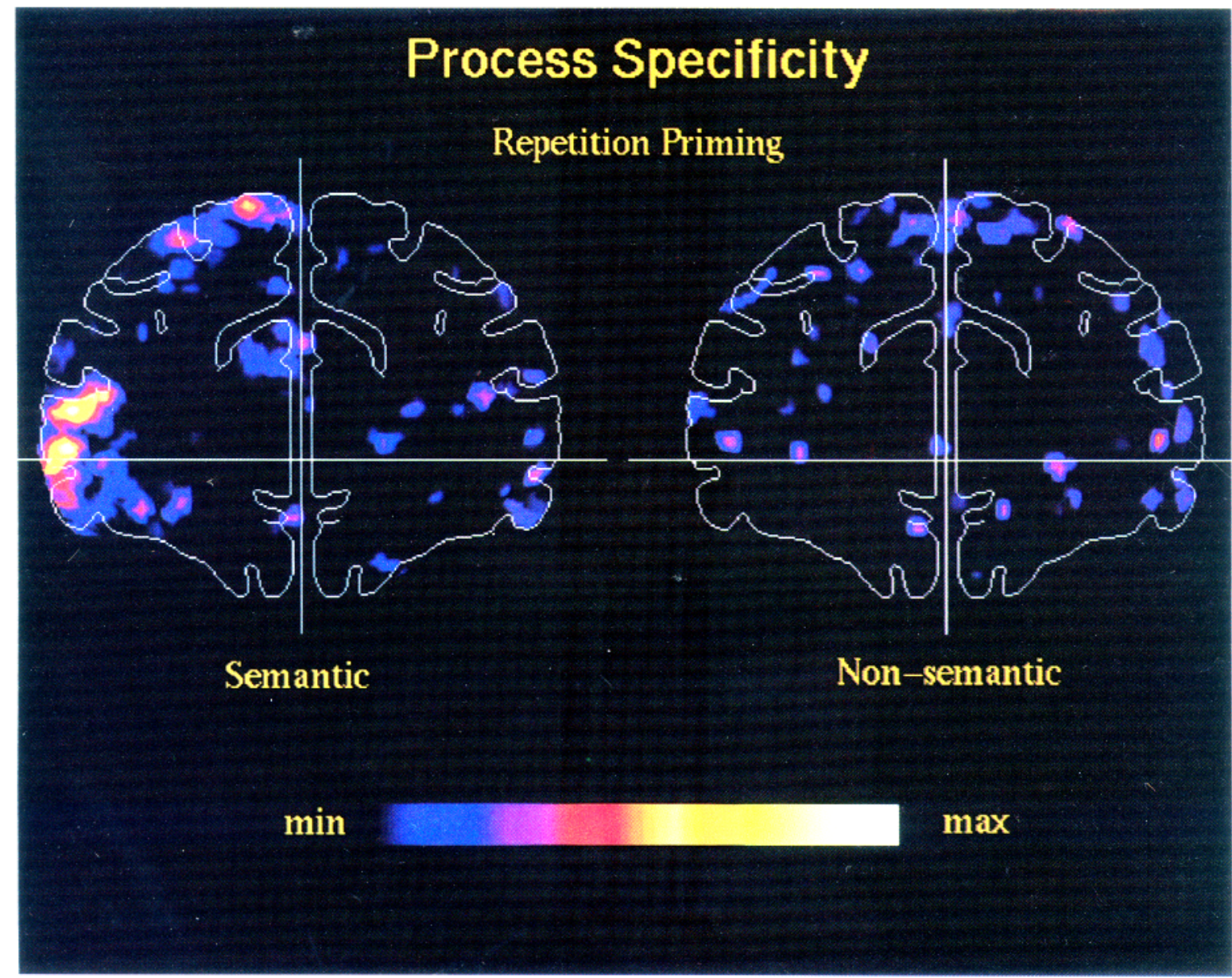


that facilitation in processing during repeated semantic encoding (Gabrieli et al., unpublished observations) reflects a process-specific change rather than a consequence of mere repeated exposure to words. There was also activation in area 8 of the LSFG in four of six subjects that showed the same pattern as the LIPC activation. This decrease during semantic reencoding reflects an implicit retrieval of the previous encoding experience with the same words.

Because facilitation of processing is correlated with decreased fMRI activation, a plausible interpretation is that repeated semantic processing requires less neuronal activity relative to initial processing. This type of decreased response to the processing of repeated stimuli has been reported at the single cell level in monkeys performing an object working memory task (Miller et al., 1993; Miller and Desimone, 1994). Decreased firing to repeated stimuli has also been observed across working memory trials, which suggests that this effect is present in both shortterm and long-term memory (Li et al., 1993). In these studies, cells in extrastriate regions of the temporal lobe fired less during repeated relative to initial stimulus presentation.

The LIPC locus of decreased activation during repeated semantic encoding in the Process Specificity Experiment was similar to the LIPC locus of increased activation during semantic encoding in the Task Difficulty Expcriment. We have found similar patterns of overlapping activation within the same subjects (Gabrieli et al., unpublished observations). The colocalization of this area suggests that a common neural network may (1) semantically encode incoming verbal information and (2) require less neural computation during the repeated semantic encoding of that information. This facilitation is displayed at a behavioral level as decreased RT, and at a neuronal level as decreased activation. A previous PET study reported similar decreases in response time and LIPC activation during practiced verb generation relative to initial verb generation with the same stimuli (Raichle et al., 1994). Raichle et al. attributed the decreased LIPC activation to enhanced verbal response selection. However, the current study found decreased LIPC activation even though no verbal response was required by the semantic task. Thus, the decreased activation may reflect more efficient access to recently processed scmantic information rather than an ease in verbal response selection per se.

In a PET study, Squire et al. (1992) reported a perceptual repetition priming response in the right occipital lobe (Marsolek et al., 1993). In that study, subjects generated words to word stems (e.g., MOT_ ) that could be completed with recently seen words (e.g., MOTEL). Normal subjects as well as patients with global anmesia show a tendency to complete word stems with previously seen words relative to other completions (e.g., MOTHER) (Warrington and Weiskrantz, 1968; Graf et al., 1984). Squire et al. (1992) reported decreased activation during priming in right occipital areas that were initially activated during a baseline condition in which subjects completed baseline (unstudied) stems (Buckner et al., 1995). The decreased LIPC activation in the current study during repeated semantic encoding can be termed semantic (conceptual) implicit retrieval, an experience-based facilitation in semantic processing that does not depend on intentional recollection (Blaxton, 1989; Keane et al., 1991; Gabrieli et al., 1995). There may be a right occipital memory system that participates in perceptual implicit memory (Squire et al., 1992; Marsolek et al., 1993; Gabrieli et al., 1995), and a left inferior prefrontal memory system that participates in semantic implicit memory (Gabrieli et al., unpublished observations). Both expressions of implicit memory are represented by decreased activation in the same brain areas involved in initial task processing.

\section{Theoretical implications}

In the current study, the Semantic Encoding Task required online access to semantic information not present in the stimulus display. The LIPC may act as a Semantic Executive System (SES) that can perform this on-line access, similar to executive systems in models of working memory (Baddeley, 1986; Goldman-Rakic, 1987). Typically, working memory models include an executive system in regions of the frontal lobe, and a shortterm buffer, or "slave system" in posterior cortical areas. In the monkey, object and spatial working memory appear to have domain-specific frontal executive systems with storage buffers in the temporal or parietal lobe, respectively (Funahashi et al., 1989; Goldman-Rakic et al., 1987; Wilson et al., 1993). In the human, functional imaging studies have suggest that spatial and verbal working memory may have frontal executive systems with storage buffers in posterior areas (Jonides et al., 1993; Paulesu et al., 1993; Cohen et al., 1994; McCarthy et al., 1994). The primary difference between models of working memory and the current model is that the SES may not be paired with a "slave system" that stores domain specific information for a short period (e.g., 5-7 sec). Instead, this system may be involved in the on-line access to information in a long-term semantic store (i.e., word meaning). The proximity to Broca's area suggests that the LIPC may be important for semantic processing during speech output. We found that increased prefrontal activation during our semantic task was lateralized to the side of speech dominlance in Wada-tested patients (Desmond et al., in press). This area, however, is functionally distinct from Broca's area: semantic processing tasks may cause activation in this area whereas lower-level verbal processes do not (Petersen et al., 1988, 1989, 1990; Abdullaev and Bechtereva, 1993).

\section{References}

Abdullaev YG, Bechtereva NP (1993) Neuronal correlate of the higherorder semantic code in human prefrontal cortex in language tasks. Int J Psychophysiol 14:167-177.

Baddeley AD (1986) Working memory. Oxford: Oxford UP.

Bandettini PA, Jesmanowicz A, Wong EC, Hyde IS (1993) Processing strategies for time-course data sets in functional MRI of the human brain. Magn Reson Med 30:161-173.

Blaxton TA (1989) Investigating dissociations among memory mea-

Figure 4. Averaged fMRI activations for four subjects in the Task Difficulty experiment and five subjects in the Process Specificity experiment. Composite images are averages of two slices from each subject $(y=+32 \mathrm{~mm}$ and $+39 \mathrm{~mm})$ are displayed on a coronal section at $\mathrm{y}=+35 \mathrm{~mm}$ (Talairach and Tournoux, 1988). Greater averaged fMRI activation during semantic encoding (Abstract/Concrete Task) relative to easy nonsemantic encoding (Uppercase/Lowercase Task) (upper row, left side) and during semantic encoding relative to difficult nonsemantic encoding (Ascending/ Descending Task) (upper row, right side). Decreased averaged fMRI activation during repeated relative to initial semantic encoding (semantic repetition priming) (lower row, left side) and during repeated relative to initial nonsemantic encoding (nonsemantic repetition priming) (lower row, right side). Left side of image corresponds to left side of brain. The color scale indicates normalized, correlation-weighted sums across subjects for each experiment. The absolute value of the color scale is the same in all slices. 
sures: support for a transfer-appropriate processing framework. J Exp Psychol Learn Mem Cognit 15:657-668.

Buckner RL, Petersen SE, Ojemann JG, Miezin FM, Squire LR, Raichle ME (1995) Functional anatomical studies of explicit and implicit memory retrieval tasks. J Neurosci 12:12-29.

Cohen JD, Forman SD, Braver TS, Casey BJ, Servan-Schreiber D, Noll DC (1994) Activation of prefrontal cortex in a non-spatial working memory task with functional MRI. Human Brain Mapping 1:293304.

Craik FIM, Lockhart RS (1972) Levels of processing: a framework for memory research. J Verb Learn Verb Behav 11:671-684.

Craik FIM, Tulving E (1975) Depth of processing and the retention of words in episodic memory. J Exp Psychol Gen 104:268-294.

Demonet JF, Chollet F, Ramsay S, Cardebat D, Nespoulous JL, Wise R, Rascol A, Frackowiak R (1992) The anatomy of phonological and semantic processing in normal subjects. Brain 115:1753-1768.

Desmond JE, Sum JM, Wagner AD, Demb JB, Shear PK, Glover GH, Gabrieli JDE, Morrell MJ (in press) Language lateralization in Wada-tested patients using functional MRI. Brain, in press.

Funahashi S, Bruce CJ, Goldman-Rakic PS (1989) Mnemonic coding of visual space in the monkey's dorsolateral prefrontal cortex. J Neurophysiol 61:331-349.

Gabrieli JDE, Fleischman DA, Keane MM, Reminger SL, Morrell F (1995) Double dissociation between memory systems underlying explicit and implicit memory in the human brain. Psychol Science 6:7682.

Goldman-Rakic PS (1987) Circuitry of primate prefrontal cortex and regulation of behavior by representational memory. In: Handbook of physiology (Plum F, Mountcastle V, eds), pp 373-417. Bcthesda, MD: American Physiological Society.

Graf P, Schacter DL (1985) Implicit and explicit memory for new associations in normal and amnesic subjects. $\mathbf{J}$ Exp Psychol Learn Mem Cognit 11:501-518.

Graf P, Squire LR, Mandler G (1984) The information that amnesic patients do not forget. J Exp Psychol Learn Mem Cognit 10:164178.

Jonides J, Smith EE, Koeppe RA, Awh E, Minoshima S, Mintun MA (1993) Spatial working memory in humans as revealed by PET. Nature 363:623-625.

Kapur S, Craik FIM, Tulving E, Wilson $\Lambda \Lambda$, Houle SH, Brown GM (1994) Neuroanatomical correlates of encoding in episodic memory: levels of processing effect. Proc Natl Acad Sci USA 91:2008-2011.

Keane MM, Gabrieli JDE, Fennema AC, Growdon JH, Corkin S (1991) Evidence for a dissociation between perceptual and conceptual priming in Alzheimer's disease. Behav Neurosci 105:326-342.

Lee AT, Glover GH, Meyer CH (in press) Discrimination of large venous vessels in time-course spiral blood-oxygen-level-dependent magnetic-resonance functional neuroimaging. Magn Reson Med, in press.

Li L, Miller EK, Desimone R (1993) The representation of stimulus familiarity in anterior inferior temporal cortex. J Neurophysiol 69: 1918-1929.
Marsolek CJ, Kosslyn SM, Squire I.R (1992) Form-specific visual priming in the right cerebral hemisphere. J Exp Psychol Learn Mem Cognit 18:492-508.

Mazoyer BM, Tzourio N, Frak V, Syrota $\Lambda$, Murayama N, Levrier O, Salamon G, Dehaene S, Cohen L, Mehler J (1993) The cortical representation of speech. J Cognit Neurosci 5:467-479.

McCarthy G, Blamire AM, Rothman DL, Gruetler R, Shulıan RG (1993) Echo-planar magnetic resonance imaging studies of frontal cortex activation during word generation in humans. Proc Natl Acad Sci USA 90:4952-4956.

McCarthy G, Blamire AM, Puce A, Nobre AC, Bloch G, Hyder F, Goldman-Rakic P, Shulman RG (1994) Functional magnetic resonance imaging of human prefrontal cortex activation during a spatial working memory task. Proc Natl Acad Sci USA 91:8690-8694.

Meyer CH, Hu BS, Nishimura DG, Macovski A (1992) Fast spiral coronary artery imaging. Magn Reson Med 28:202.

Miller EK, Desimone R (1994) Parallel neuronal mechanisms for shortterm memory. Science 263:520-522.

Miller EK, Li L, Desimone R (1993) Activity of neurons in anterior inferior temporal cortex during a short-term memory task. J Neurosci 12:1460-1478.

Noll DC, Cohen JD, Meyer CH, Schneider W (in press) Spiral k-space MRI of cortical activation. J Magn Reson Imag, in press.

Paulesu E, Frith CD, Frackowiak RSJ (1993) The neural correlates of the verbal component of working memory. Nature 362:342-345.

Petersen SE, Fox PT, Posner MI, Mintun M, Raichle ME (1988) Positron emission tomographic studies of the cortical anatomy of single word processing. Nature 331:585-589.

Petersen SE, Fox PT, Posner MI, Mintun M, Raichle ME (1989) Positron emission tomographic studies of the processing of single words. J Cognit Neurosci 1:153-170.

Petersen SE, Fox PT, Snyder AZ, Raichle ME (1990) Activation of extrastriate and frontal cortical areas by visual words and word-like stimuli. Science 249:1037-1041.

Raichle ME, Fiez JA, Videen TO, MacLeod AK, Pardo JV, Fox PE, Petersen SE (1994) Practice-related changes in human brain functional anatomy during nonmotor learning. Cereb Cortex 4:8-26.

Squire LR, Ojemann JG, Miezin FM, Petersen SE, Videen TO, Raichle ME (1992) Activation of the hippocampus in normal humans: A functional anatomical study of memory. Proc Natl Acad Sci USA 89: $18.37-1841$

Talairach J, Tournoux P (1988) Co-planar stereotaxic atlas of the human brain. Stuttgart: Thieme.

Tulving E, Kapur S, Craik FIM, Moscovitch M, Houle S (1994) Hemispheric encoding/retrieval asymmetry in episodic memory: positron emission tomography findings. Proc Natl Acad Sci USA 91:20162020.

Warrington EK, Weiskrantz L (1968) New method of testing long-term retention with special reference to amnesic patients. Nature 217:972974.

Wilson FAW, O'Scalaidhe SP, Goldman-Rakic PS (1993) Dissociation of object and spatial processing in primate prefrontal cortex. Science 260:1955-1958. 